ARTIGO ORIGINAL

ORIGINAL ARTICLE

\title{
Modelo de impacto orçamentário da aplicação do single embryo transfer consecutivo comparado com o double embryo transfer em tratamentos de fertilização in vitro no Sistema de Saúde Suplementar
}

\author{
Budget impact model of the use of consecutive single embryo \\ transfers compared to double embryo transfer in in vitro \\ fertilization procedures in the Brazilian Private Healthcare System \\ Eli Lakryc ${ }^{1}$, Rodrigo Rodrigues', Danylo Panovyk¹, Pedro Monteleone ${ }^{2,3}$, Eduardo Motta ${ }^{3,4}$ \\ DOI: 10.21115/JBES.v11.n3.p231-43
}

\section{Palavras-chave:}

single embryo transfer, double embryo transfer, Brasil, Sistema de Saúde Suplementar, infertilidade, custos

\section{Keywords:}

single embryo transfer, double embryo transfer, Brazil, Private Healthcare System, infertility, costs

\section{RESUMO}

Objetivo: Estimar o impacto orçamentário em cinco anos da incorporação da técnica de SET (single embryo transfer) sequencial no Sistema de Saúde Suplementar brasileiro (SSSB). Métodos: Foram consideradas taxas de nascidos vivos de $41 \%$ e de gemelaridade de $2 \%$ e 30\% para SET e DET (double transfer embryo), respectivamente. Os custos dos procedimentos foram, em sua maioria, obtidos das tabelas SIMPRO, CBHPM e CMED. Para a estimativa da população elegível, foram consideradas mulheres de 20 a 35 anos registradas no Datasus e taxas de infertilidade e de uso de fertilização in vitro (FIV) da literatura. Estimou-se em 20\% e $80 \%$ o uso de SET sequencial e DET, respectivamente, com incorporação da primeira em 45\%,50\%, 55\%,65\% e 75\% dos casos nos cinco anos subsequentes. Resultados: Considerando cenários com reembolso de um a dois ciclos de SET sequencial e inclusão ou não do valor dos medicamentos, o impacto orçamentário em cinco anos seria entre $\mathrm{R} \$ 10.231 .387$ e R\$ 16.123.874 em operadoras de grande porte, R\$ 1.054.174 e R\$ 1.661.297 em operadoras de médio porte e $\mathrm{R} \$ 173.700$ e $\mathrm{R} \$ 273.738$ em operadoras de pequeno porte. Em cenário com aumento progressivo do uso de SET sequencial sem alteração no modelo de reembolso atual, foi estimada economia de R\$ 59.319.276 para o SSSB em cinco anos. Conclusões: O uso de SET sequencial (vs. DET) em procedimentos de FIV é potencialmente menos oneroso para o SSSB referente a custos de pré-natal, parto e complicações. Em cenário de incorporação progressiva, o custo incremental foi estimado em $\mathrm{R} \$$ 8-13 mil/paciente.

\begin{abstract}
Objective: To estimate the five-year budget impact of incorporating the sequential single embryo transfer (SET) technique into the Brazilian Supplementary Healthcare System (BSHS). Methods: Live birth rates of $41 \%$ and multiple pregnancy rates of $2 \%$ and $30 \%$ were considered for SET and DET (double transfer embryo), respectively. The costs of the procedures were mostly obtained from e SIMPRO, CBHPM and CMED tables. To estimate the eligible population, we considered women aged 20-35 years registered in DATASUS, and infertility and in vitro fertilization (IVF) rates from the literature. The use of sequential SET and DET was estimated as 20\% and $80 \%$, respectively, with incorporation of the first in $45 \%, 50 \%, 55 \%, 65 \%$ and $75 \%$ of cases in the subsequent five years. Results: Considering scenarios with reimbursement of 1 to 2 cycles of sequential SET and inclusion or not of the drugs in the reimbursement, the budget impact in five years would be between BRL 10,231,387-16,123,874 in large operators, BRL 1,054,174-1,661,297 in midsize operators and BRL $173,700-273,738$ in small operators. In a scenario of progressive increase of the use of sequential
\end{abstract}

Recebido em: 29/10/2019. Aprovado para publicação em: 13/11/2019

1. Ferring Pharmaceuticals, São Paulo, SP, Brasil.

2. Clínica Monteleone, São Paulo, SP, Brasil.

3. Departamento de Ginecologia, Universidade de São Paulo, São Paulo, SP, Brasil.

4. Clínica Huntington, São Paulo, SP, Brasil.

Auxílios: Este estudo teve suporte financeiro da Ferring Pharmaceuticals para a sua realização.

Conflito de interesses: Alguns dos autores são funcionários da Ferring Pharmaceuticals, São Paulo, SP, Brasil.

Autor correspondente: Eli Lakryc. Praça São Marcos, 624, Vila Ida, São Paulo, SP, Brasil. CEP: 05455-050.

Telefone: +55 (11) 97086-6533. E-mail: Eli.Lakryc@ferring.com 
SET with no change in the current reimbursement model, we estimated a saving of BRL 59,319,276 for the BSHS in five years. Conclusions: The use of sequential SET (Vs. DET) in IVF procedures is potentially less costly for BSHS for prenatal, child-birth and complication costs. In the scenario of progressive incorporation and full reimbursement of the procedure, the incremental cost was estimated at BRL 8-13 thousands/patient.

\section{Introdução}

A infertilidade (Classificação Internacional de Doenças v.10 N97 [Infertilidade Feminina] ou N46 [Infertilidade Masculina]) é uma patologia considerada um problema de saúde pública pela Organização Mundial da Saúde (OMS). É definida como a incapacidade de conceber após a realização de relações sexuais regulares sem contracepção pelo período de um ano (Cui, 2010).

No Brasil, estimou-se uma taxa de infertilidade entre $8 \%$ e 15\% em 2010, sendo o fator tubário o principal para a infertilidade associada a mulher (Pantoja \& Fernandes, 2015)

As técnicas de reprodução assistida (TRAs) foram introduzidas na década de 1970 e, desde então, o número de procedimentos vem aumentando no Brasil e em outras regiões do mundo (Correa \& Loyola, 2015; Kushnir et al., 2017). Segundo o relatório do sistema nacional de produção de embriões (SisEmbrio), no Brasil, 43.098 ciclos de fertilização in vitro foram realizados em 2018 (205,2 ciclos por milhão de habitantes) e 36.307 em 2017 (172,9 ciclos por milhão de habitantes) (Anvisa, 2019).

Mesmo com esse crescimento de aproximadamente 19\% no ano de 2018, a prática de TRAs no Brasil é relativamente reduzida. Em comparação, a Argentina e a Alemanha realizaram, em 2016, 472 e 1.216 ciclos por milhão de habitantes, respectivamente (Blumenauer et al., 2018; Zegers-Hochschild et al., 2019).

Existem diversas TRAs que diferem entre si de acordo com sua complexidade, taxas de sucesso e custos do tratamento. Entre as TRAs de baixa complexidade, podemos citar a relação sexual programada e a inseminação intrauterina, enquanto a fertilização in vitro corresponde à TRA de alta complexidade (Farquhar \& Marjoribanks, 2018).

Os tratamentos da infertilidade masculina e feminina têm cobertura obrigatória no Sistema de Saúde Suplementar no Brasil, desde que listados no rol mínimo de cobertura de procedimentos das operadoras de planos de saúde, na segmentação contratada (ANS, 2019). Entretanto, segundo descrito na Lei dos Planos de Saúde no 9.656/1998, o plano-referência de assistência à saúde não tem obrigatoriedade da cobertura de procedimentos de TRA, apesar de essa mesma lei estabelecer a obrigatoriedade de cobertura de todas as doenças reconhecidas pelo Código Internacional de Doenças (CID). Ainda, segundo descrito posteriormente na Lei Federal no 11.935/2009, os planos e seguros privados de assistência à saúde no país devem cobrir os casos de planejamento familiar, incluindo a concepção (Brasil, 1988, 2009). No entanto, segundo a mais recente resolução normativa RN n० 428 da
ANS, o procedimento de TRA é listado como uma exclusão assistencial do plano-referência (Art. 20, § 10, III) (ANS, 2017).

Ou seja, até o presente momento não está prevista a cobertura de TRAs no rol mínimo de procedimentos das operadoras de planos de saúde (ANS, 2017).

Segundo um levantamento on-line realizado em oito Tribunais de Justiça do país (São Paulo, Rio Grande do Sul, Rio de Janeiro, Minas Gerais, Distrito Federal, Mato Grosso do Sul, Bahia e Pernambuco), o número de ações de casais brasileiros que recorreram à judicialização para cobertura de TRAs pelas operadoras de saúde aumentou de 8 para 20 entre 2015 e 2016. Em cerca de 78\% desses casos, a decisão foi favorável aos casais (Collucci, 2017).

Por outro lado, os custos relacionados à gestação, como o acompanhamento pré-natal com a realização de consultas, exames laboratoriais e de ultrassonografia, ao parto e suas complicações e à internação do recém-nascido, que muitas vezes pode requerer uma unidade de terapia intensiva (UTI), são obrigatoriamente cobertos pelas operadoras de saúde (Brasil, 1998) (Figura 1).

Nos Estados Unidos, a introdução das TRAs levou a um aumento nos nascimentos múltiplos, o que representa risco

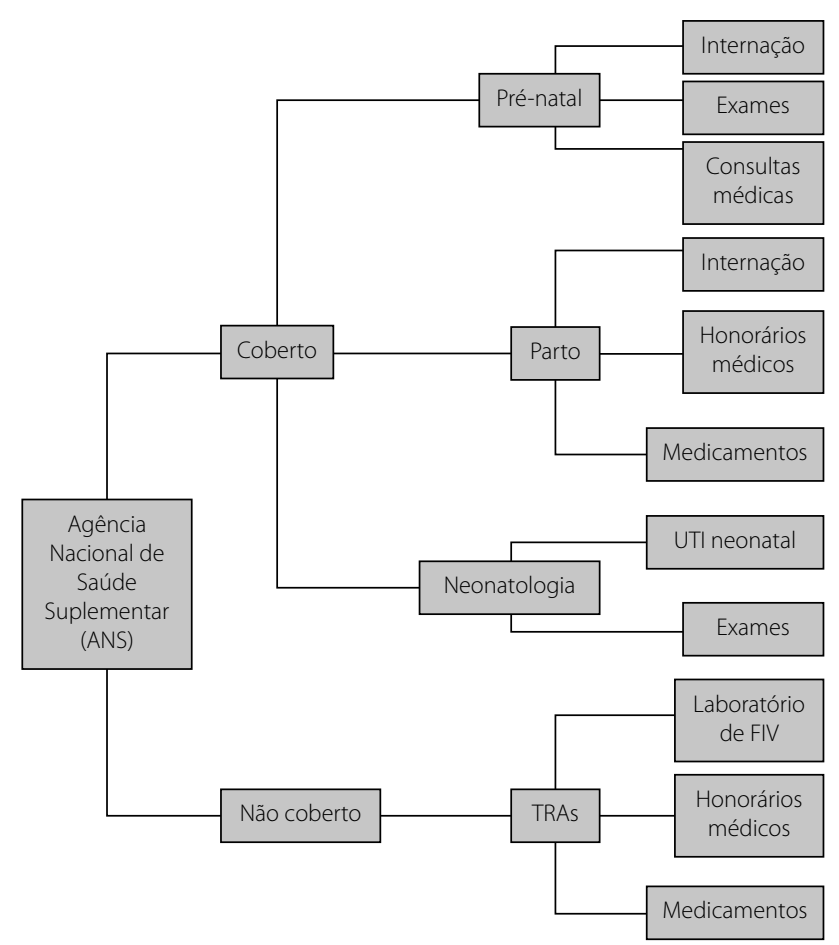

Figura 1. Etapas de planejamento familiar com reembolso ou não pelas operadoras de saúde.

UTI: unidade de terapia intensiva; TRAs: técnicas de reprodução assistida. 
para a saúde da gestante (por exemplo: diabetes gestacional, hipertensão induzida pela gravidez, parto cesáreo e hemorragia) e do recém-nascido (por exemplo: elevado risco de prematuridade, baixo peso ao nascer, anomalias congênitas, paralisia cerebral, autismo e óbito) (Crawford et al., 2016). Ainda, as implicações econômicas para os serviços de saúde resultantes do aumento de nascimentos múltiplos são consideráveis, uma vez que os nascidos pré-termo apresentam taxas significativamente maiores de morbimortalidade, e os custos são inversamente relacionadas à idade gestacional ao nascimento (Henderson et al., 2004).

Uma estratégia efetiva para reduzir a quantidade de nascimentos múltiplos observada após a TRA consiste na contenção do número de embriões transferidos durante um ciclo de tratamento (Crawford et al., 2016). A probabilidade de gravidez múltipla em ciclos com transferência de dois embriões simultaneamente é de $30 \%$, enquanto em transferência de um único embrião é de $2 \%$ (Monteleone et al., 2018).

Segundo a mais recente resolução do Conselho Federal de Medicina ( $n^{\circ}$ 2.168/2017), mulheres com idade inferior ou igual a 35 anos têm o limite máximo de até dois embriões que podem ser transferidos simultaneamente durante um tratamento de fertilização in vitro. Assim, para essa faixa etária, as opções são a realização de transferência de um único embrião (single embryo transfer - SET) ou dois embriões (double embryo transfer - DET) (Conselho Federal de Medicina, 2017).

Estudos comparativos demonstram que a técnica de utilizar sequencialmente dois SETs, isto é, a transferência de um único embrião (SET) com o congelamento dos demais embriões excedentes e posteriormente a transferência de outro embrião único (SET) descongelado, caso o primeiro ciclo não resulte em um nascido vivo, proporciona consideráveis vantagens, mantendo taxas de nascidos vivos cumulativos similares, quando comparada com a técnica de transferir dois embriões simultaneamente (DET) Figura 2 (Pandian et al., 2013; McLernon et al., 2010).

Assim, a técnica de SET sequencial (SET + SET) não só consegue minimizar a probabilidade de gravidez múltipla, quando comparada à técnica de DET, mas também tem o potencial de reduzir o risco de complicações da gestação e minimizar a chance de prematuridade extrema, baixo peso ao nascer e complicações associadas, preservando as taxas de gravidez (Cutting, 2018; Grady et al., 2012; Meldrum et al., 2018). Diversos estudos econômicos foram produzidos para estimar a custo-efetividade das técnicas de SET e DET, e o impacto econômico das gestações múltiplas no sistema de saúde (Carpinello et al., 2016; Hernandez Torres et al., 2015; Lee et al., 2016). No entanto, não há uma concordância universal sobre o tema, isso porque as análises produzidas são fortemente influenciadas pelos procedimentos médicos praticados em cada localização geográfica, tal como custo da intervenção médica e medicação necessária.

Vale ainda salientar que, atualmente, os procedimentos são integralmente custeados pela população usuária das TRAs, logo a transferência de dois embriões por ciclo é escoIhida por apresentar melhores resultados imediatos, não se considerando neste momento os custos e as complicações da gestação dupla, da prematuridade ou mesmo das demandas a longo prazo, que necessariamente serão financiadas pela saúde suplementar ou sistema público de saúde.

Assim, este estudo tem por objetivo avaliar a diferença dos custos entre a técnica de SET sequencial (transferência de um embrião e depois outro congelado, caso o primeiro ciclo não resulte em um nascido vivo) e DET (transferência de dois embriões simultaneamente), bem como estimar o impacto orçamentário em cinco anos da incorporação da técnica de SET sequencial no Sistema de Saúde Suplementar brasileiro (Woods et al., 2017).

\section{Métodos}

Atualmente no Brasil, o procedimento de fertilização in vitro, quer seja com a utilização de SET ou DET, é amplamente utilizado, porém não é reembolsado pelo Sistema de Saúde Suplementar. No entanto, os custos relacionados ao acompanhamento pré-natal, tais como consultas médicas, exames laboratoriais, ultrassonografias obstétricas, eventual internação hospitalar da gestante, bem como ao parto e cuidados ao(s) recém-nascido(s) são cobertos pelos planos de saúde (Brasil, 1998).

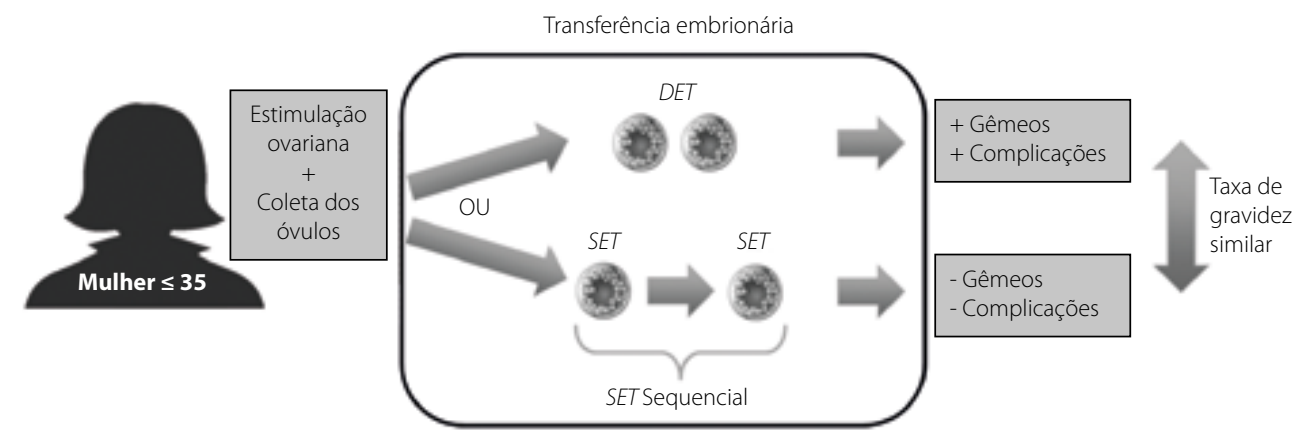

Figura 2. Diferença entre SET sequencial e DET.

SET: single embryo transfer; DET: double transfer embryo. 
Assim, o modelo de impacto orçamentário avaliou o impacto da incorporação da técnica de SET sequencial (transferência de um embrião e depois outro congelado, caso o primeiro ciclo não resulte em um nascido vivo) comparativamente à transferência simultânea de dois embriões (DET) em população elegível ao tratamento com fertilização in vitro, no Sistema de Saúde Suplementar brasileiro.

O desenvolvimento desse modelo seguiu as orientações das diretrizes da International Society for Pharmacoeconomics and Outcomes Research (ISPOR) e do Ministério da Saúde (Ministério da Saúde, 2014a; Sullivan et al., 2014).

\section{Parâmetros clínicos}

Resultados de uma revisão sistemática da literatura sugerem que a taxa cumulativa de nascidos vivos a partir da técnica de SET sequencial (transferência de um embrião e depois outro congelado, caso o primeiro ciclo não resulte em um nascido vivo) ou DET (transferência simultânea de dois embriões) não apresenta diferenças significativas (Grady et al., 2012). Dessa forma, o modelo considerou uma taxa de nascidos vivos de $41 \%$, em mulheres com idade igual ou inferior a 35 anos, para ambas as técnicas (Mehta et al., 2018; Mukherjee \& Khastgir, 2018; SART, 2018). Por outro lado, o procedimento de SET sequencial apresenta uma taxa de gemelaridade de $2 \%$, enquanto no procedimento DET essa taxa é de 30\% (Monteleone et al., 2018). A Figura 3 sumariza a estrutura da árvore de decisão adotada no modelo e os parâmetros clínicos utilizados.

\section{Custos}

Custos diretos referentes ao procedimento de fertilização in vitro, ao acompanhamento pré-natal, ao parto e ao recém-nascido(s), e a possíveis complicações foram considerados. No procedimento de fertilização in vitro, foram levados em consideração os custos do laboratório de embriologia, bem como exames e honorários médicos.

Foram incluídos nos custos de pré-natal consultas médicas, exames de ultrassonografia obstétrica e exames laboratoriais. Para o parto, deduziram-se os materiais, honorários médicos, medicamentos e exames. Por fim, nas complicações de curto prazo, utilizou-se o custo da diária na UTI neonatal.

Foi necessário levantar a frequência de uso dos recursos, por exemplo, o número de exames realizados, para gestações de feto único ou múltiplos. Foram feitas médias ponderadas dos dados divulgados pela National Perinatal Epidemiology Unit. Os dados de frequência encontrados foram utilizados para realizar o custeio (Tabela 1).

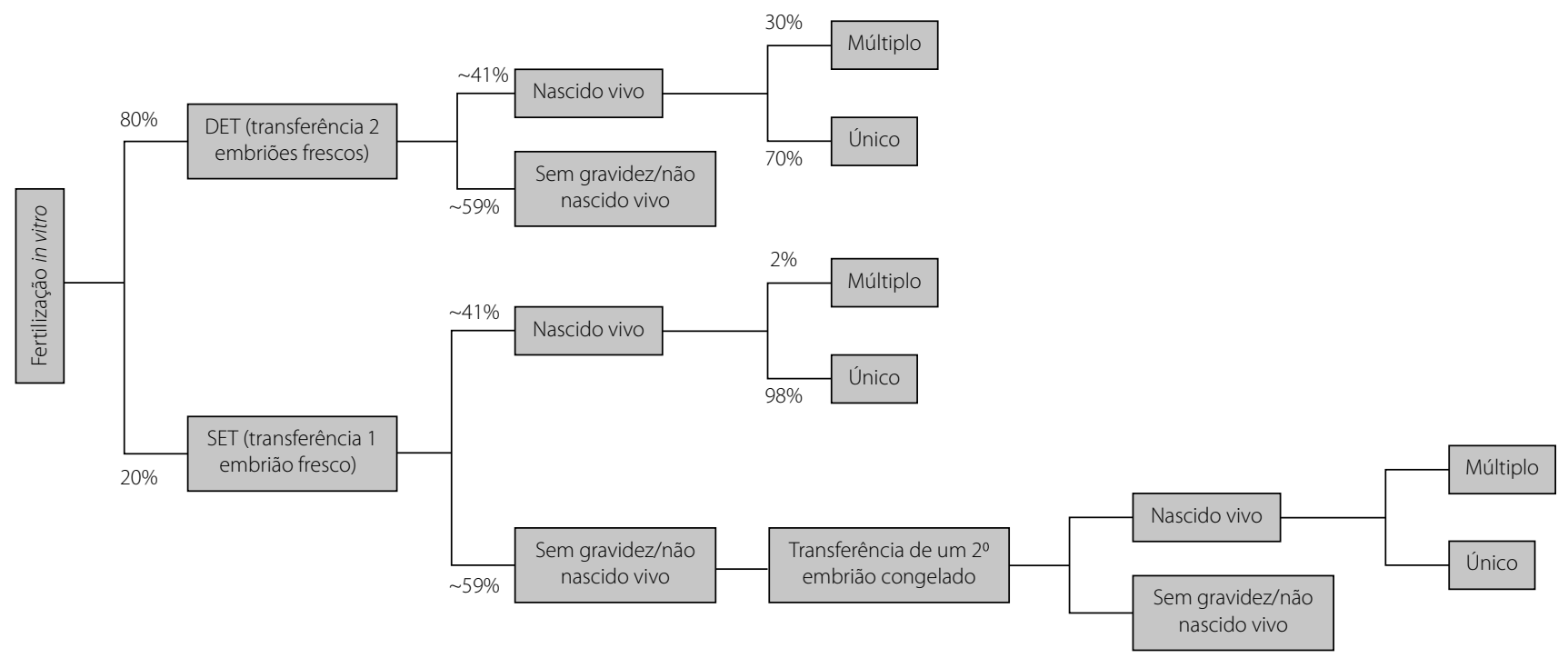

Figura 3. Fluxograma dos parâmetros clínicos utilizados.

* A taxa de $41 \%$ refere-se à taxa real de nascidos vivos no primeiro ciclo de fertilização in vitro. Note que no caso de SET sequencial (SET + SET) essa taxa é aplicada à população elegível.

Tabela 1. Diferença de frequência de uso de recursos quando múltiplo e único

\begin{tabular}{lccc}
\hline Tipo & Recurso & Múltiplo & Único \\
\hline \multirow{2}{*}{ Pré-natal } & Número de exames & 5,61 & 2,92 \\
\cline { 2 - 5 } & Número de consultas & 13 & 9 \\
\hline Complicações & Número médio de dias internado na UTI & 10,66 & 5,9 \\
\hline Parto & Número médio de diárias de internação da mãe e recém-nascido pós-parto & 3,75 & 2 \\
\hline
\end{tabular}


Preços foram obtidos a partir da tabela de preço Simpro de janeiro/2019 para os materiais, Classificação Brasileira Hierarquizada de Procedimentos Médicos (CBHPM) de 2016 para os procedimentos e Câmara de Regulação do Mercado de Medicamentos (CMED) de janeiro/2019 para medicamentos.

Os custos da diária da UTI neonatal, assim como os custos dos procedimentos de SET sequencial e DET, incluindo medicamentos, procedimentos e honorários médicos não estão disponíveis na tabela de preços e não foram encontrados dados na literatura. Assim, consultou-se um especialista em fertilização in vitro, ativo em clínica no mercado, para estimar esses custos. Dada a limitação desses dados de custo, estes foram avaliados na análise de sensibilidade determinística.

\section{População elegível}

A população elegível considerada foram mulheres de 20 a 35 anos que precisam da fertilização in vitro caso queiram engravidar. A faixa de idade de 20 a 35 anos foi escolhida por conta das orientações da American Society for Reproductive Medicine, que recomenda a técnica de SET para mulheres mais jovens e, portanto, com melhor prognóstico. É importante destacar que, pela regulação médica brasileira - Resolução do ConseIho Federal de Medicina no 2.168/2017 -, para mulheres com idade igual ou inferior a 35 anos, é permitida a transferência de no máximo dois embriões simultaneamente (Conselho Federal de Medicina, 2017).

A Figura 4 mostra o fluxograma da população elegível ao procedimento de fertilização in vitro.

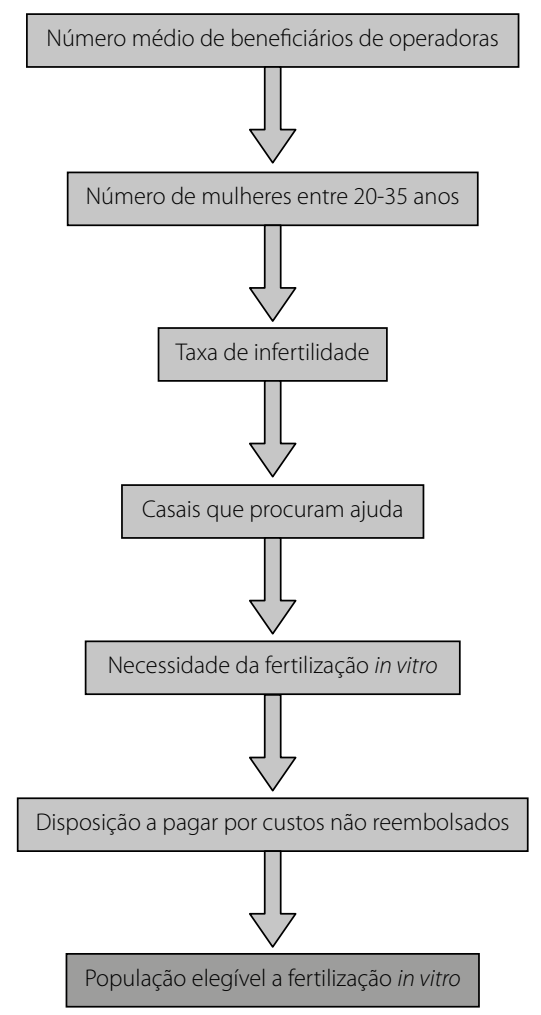

Figura 4. Fluxograma da população elegível.
A estimativa da população elegível ao tratamento da fertilização in vitro foi realizada a partir do método epidemiológico, no qual os dados de incidência e/ou prevalência da condição estudada são aplicados nos dados populacionais das perspectivas de interesse (Ministério da Saúde, 2014a).

A estimativa do número de beneficiários do Sistema de Saúde Suplementar para os anos de 2021 a 2025 foi feita a partir dos dados publicados pela Agência Nacional de Saúde Suplementar (ANS). Para cada porte de operadora de saúde, estimou-se o número médio de beneficiários que se enquadram em cada categoria. Em 2018, os números médios de beneficiários nas operadoras de grande, médio e pequeno porte foram 455.435, 46.925 e 7.732 pessoas, respectivamente (ANS, 2017). Uma vez que a taxa de crescimento variou de $-3,2 \%$ (dezembro/2016) até 0,3\% (dezembro/2018), adotou-se uma taxa de crescimento de $0,82 \%$ do número de beneficiários que acompanha a estimativa de crescimento populacional divulgada pelo Instituto Brasileiro de Geografia e Estatística (IBGE) (ANS, 2018; IBGE, 2018).

Para estimar o número de mulheres entre 20 e 35 anos que seriam elegíveis ao procedimento, utilizou-se a distribuição dos dados populacionais completos do Brasil disponíveis no Datasus, que aponta que cerca de 13,06\% da população é de mulheres na faixa etária de interesse. A taxa de infertilidade aplicada foi de $9 \%$ de mulheres, entre as quais se considerou que 51,2\% procuram ajuda para engravidar (Boivin et al., 2007) e que 50\% têm necessidade de uso da técnica de fertilização in vitro de alta complexidade, uma vez que existem outros métodos de fertilização (Datasus, 2018; ESHRE, 2001).

No primeiro ano do impacto orçamentário, ou seja, 2021, foi considerado que todas as mulheres elegíveis realizariam um procedimento de fertilização in vitro (DET ou SET sequencial, divisão de acordo com market share). Nos anos seguintes, desconsideraram-se aquelas que já obtiveram um filho nascido vivo e aquelas que não retornariam para outra tentativa por alguma razão. A taxa de mulheres que não retornam é de cerca de $17,5 \%$ para a segunda tentativa e $27 \%$ não retornam para uma terceira tentativa. Assumiu-se que no quarto e no quinto ciclo essa taxa de $27 \%$ se mantém constante (Malizia et al., 2009; Klenov et al., 2017). Para se cogitar um cenário mais conservador, assumiu-se que, em média, uma mulher tentaria engravidar em cinco ciclos em um horizonte temporal de cinco anos. A taxa cumulativa de nascidos vivos considerada foi de $41 \%$ após o primeiro ciclo, $60 \%$ após o segundo ciclo e $72 \%$ após o terceiro ciclo (SART, 2018). Por limitações de dados na literatura, assumiu-se que essa taxa é de 78\% e $82 \%$ no quarto e no quinto ciclo. A taxa real calculada de nascidos vivos nos ciclos 1, 2, 3, 4 e 5 foi de 41\%, 32\%, 30\%, 21\% e $18 \%$, respectivamente (SART, 2018).

Uma vez que o custo dos medicamentos para cada procedimento de fertilização in vitro fica em torno dos $\mathrm{R} \$ 5.000,00$ e esse custo não é reembolsado pela ANS atualmente, considerou-se que cerca de $30 \%$ da população ele- 
gível teria disposição para pagar por esse custo. Essa taxa foi calculada baseada na distribuição de renda informada pelo IBGE. Utilizou-se a premissa de que os casais estariam dispostos a pagar até $28 \%$ da renda anual do casal pelos custos do procedimento (IBGE, 2017). Considerando o custo do procedimento de SET sequencial ou DET (honorários, medicamentos e procedimento) igual a $R \$ 15.000$, obteve-se que a renda anual do casal deveria ser de, no mínimo, $\mathrm{R} \$ 53.904$, equivalente ao rendimento familiar mensal de $R \$ 4.492$ (classe média a alta).

\section{Market share}

No cenário atual, considerou-se que o SET sequencial é utilizado por 20\% das mulheres e $80 \%$ utilizam o DET. Embora, o custo do procedimento em si não seja reembolsado, atualmente os custos do pré-natal, parto e complicações são reembolsados.

No cenário proposto, o market share foi considerado uma incorporação progressiva de SET sequencial, com $45 \%$ no primeiro ano, 50\% no segundo 55\% no terceiro, $65 \%$ no quarto e $75 \%$ no quinto ano.

\section{Descrição do cenário $A$}

No cenário $A$, considerado como o cenário-base, um único SET sequencial seria reembolsado, com custo de R\$10.000, referente a $\mathrm{R} \$ 5.000$ de procedimento e $\mathrm{R} \$ 5.000$ de honorários médicos, sem reembolso dos custos dos medicamentos, ou seja, a paciente arcará com esse custo (out-of pocket). O segundo, o terceiro, o quarto e o quinto procedimento de fertilização in vitro seriam o DET, não reembolsado pelo sistema de saúde, com reembolso apenas dos custos de pré-natal, parto e complicações.

\section{Descrição do cenário $B$}

No cenário $B$, foi realizada a análise de impacto orçamentário sob a premissa de que a paciente realizará SET sequencial, reembolsável pela operadora de saúde. O custo de cada SET sequencial é de $\mathrm{R} \$ 10.000$ referente aos custos de $\mathrm{R} \$ 5.000$ de procedimento e $R \$ 5.000$ de honorários médicos (opinião de especialista) e sem o reembolso dos custos dos medicamentos. Um segundo SET sequencial seria realizado para as pacientes elegíveis (aquelas que não obtiveram um nascido vivo e que retornariam para outra tentativa), com o mesmo preço e condições do primeiro SET sequencial. O terceiro, o quarto e o quinto procedimento de fertilidade para as pacientes elegíveis seriam o DET, no qual o custo do procedimento em si não é reembolsado, porém os custos de pré-natal, parto e complicações são reembolsados.

\section{Descrição do cenário $C$}

No cenário $C$, um único procedimento de SET sequencial seria reembolsado pela ANS. O custo desse SET sequencial é de R\$ 15.000, referente a R\$ 5.000 de procedimentos, $\mathrm{R} \$ 5.000$ de honorários médicos e $\mathrm{R} \$ 5.000$ de medicamento, que, diferentemente dos cenários $A$ e $B$, seria reembolsado pela ANS. O segundo, o terceiro, o quarto e o quinto procedimento de fertilização in vitro seriam o DET, não reembolsado pelo sistema de saúde, com reembolso apenas dos custos de pré-natal, parto e complicações.

\section{Descrição do cenário $D$}

Foi realizada uma comparação direta dos custos reembolsados atualmente pela ANS do DET versus SET sequencial. Portanto, compararam-se os custos de pré-natal, parto e complicações do SET sequencial versus esses mesmos custos para o DET, nos cenários de market share atual e proposto. Essa comparação foi feita por meio da uma análise de impacto orçamentário de aumento do uso do SET sequencial e diminuição do uso do DET no mercado ao longo cinco próximos anos (2021-2025) em ambos os cenários, atual e proposto, sem considerar os custos dos procedimentos de SET sequencial ou DET.

A Tabela 2 sumariza os cenários A, B, C e D utilizados nesta análise.

\section{Projeção do crescimento e utilização da fertilização in vitro}

Com o objetivo de validar o modelo elaborado que foi baseado em dados epidemiológicos, foi realizada uma estimativa de utilização da técnica de fertilização in vitro, considerando um crescimento anual e constante de $20 \%$. Este último valor foi extrapolado com base no número de ciclos de fertilização realizados no Brasil entre 2017 e 2018 (Anvisa, 2019). A Figura 5 (Anvisa, 2019; Zegers-Hochschild et al., 2019; Malizia et al., 2009) sumariza os cálculos realizados.

\section{Análise de sensibilidade}

Uma análise de sensibilidade determinística univariada foi realizada a fim de avaliar a influência da incerteza e variabi-

Tabela 2. Descrição dos cenários utilizados

\begin{tabular}{lcccc}
\hline Ano & Cenário A & Cenário B & Cenário C & Cenário D \\
\hline Ciclo 1 & SET Seq (R\$ 10.000) & SET Seq (R\$ 10.000) & SET Seq (R\$ 15.000) & SET Seq (não reembolsado) \\
\hline Ciclo 2 & DET (não reembolsado) & SET Seq (R\$ 10.000) & DET (não reembolsado) & SET Seq (não reembolsado) \\
\hline Ciclo 3 & DET (não reembolsado) & DET (não reembolsado) & DET (não reembolsado) & DET (não reembolsado) \\
\hline Ciclo 4 & DET (não reembolsado) & DET (não reembolsado) & DET (não reembolsado) & DET (não reembolsado) \\
\hline Ciclo 5 & DET (não reembolsado) & DET (não reembolsado) & DET (não reembolsado) & DET (não reembolsado) \\
\hline
\end{tabular}

* Não reembolsados: apenas o procedimento em si não é reembolsado, mas são considerados os custos de pré-natal, parto e complicações. 
lidade dos parâmetros utilizados no modelo nos resultados. As faixas de variação dos parâmetros foram baseadas em dados da literatura ou, quando não disponíveis, uma variação de $\pm 20 \%$ foi utilizada. Valores utilizados na análise podem ser encontrados na Tabela 3.

\section{Resultados}

\section{Estimativa de pacientes}

O número médio de beneficiários e o número total de muIheres entre 20 e 35 anos por porte de operadora são apresentados na Tabela 4.
O número estimado de pacientes elegíveis no primeiro ano para a realização de procedimento de fertilização in vitro é, no total, 407 em uma operadora de grande porte, 42 em uma de médio porte e 7 em uma de pequeno porte. Esses últimos referem-se ao caso em que 30\% das pacientes estariam dispostas a pagar pelos medicamentos não reembolsados pelo sistema de saúde suplementar. 0 número estimado de pacientes elegíveis ao procedimento anualmente está descrito na Tabela 5, e o número de pacientes que recorreram ao SET sequencial em cinco anos está descrito na Tabela 6.

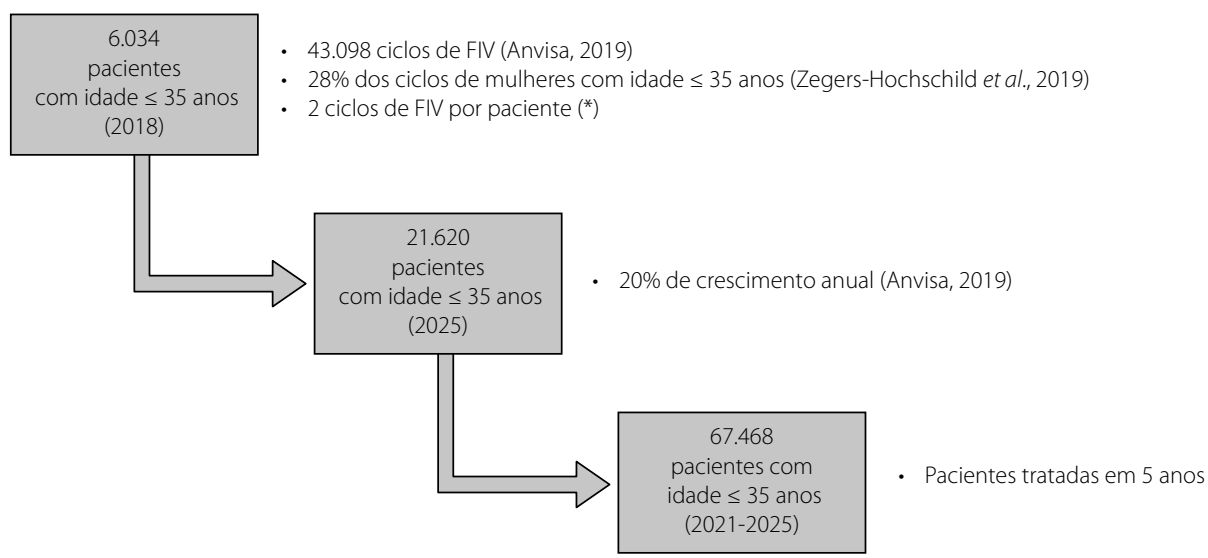

Figura 5. Estimativa do número de pacientes que utilizará fertilização in vitro (FIV) no período entre 2021 e 2025 se ocorrer um crescimento anual de $20 \%$

* Ciclos de FIV realizados por paciente estimados a partir do modelo.

Tabela 3. Variação dos parâmetros na análise de sensibilidade determinística

\begin{tabular}{|c|c|c|c|}
\hline Parâmetro & Mínimo & Valor-base & Máximo \\
\hline Taxa de infertilidade & $7,4 \%$ & $9, \%$ & $15 \%$ \\
\hline Casais que procuram ajuda & $27 \%$ & $51 \%$ & $74,1 \%$ \\
\hline Custo de laboratório & $R \$ 3.000,00$ & $\mathrm{R} \$ 5.000,00$ & $\mathrm{R} \$ 8.000,00$ \\
\hline Custo de honorários médicos & $R \$ 3.000,00$ & $\mathrm{R} \$ 5.000,00$ & $R \$ 10.000,00$ \\
\hline Taxa de gêmeos SET & $1 \%$ & $2 \%$ & $4 \%$ \\
\hline Taxa de gêmeos DET & $24 \%$ & $30 \%$ & $47 \%$ \\
\hline Taxa de crescimento populacional & $-3,20 \%$ & $0,82 \%$ & $5,60 \%$ \\
\hline Número de beneficiários & 37.902 .336 & 47.377 .920 & 56.853 .504 \\
\hline Ciclo 1 - nascidos vivos & $32,8 \%$ & $41,0 \%$ & $49,2 \%$ \\
\hline Ciclo 2 - nascidos vivos & $25,8 \%$ & $32,20 \%$ & $38,54 \%$ \\
\hline Ciclo 1 - não retorna para continuação & $14,0 \%$ & $17,5 \%$ & $21,0 \%$ \\
\hline Ciclo 2 - não retorna para continuação & $21,6 \%$ & $27,0 \%$ & $32,4 \%$ \\
\hline Custo do parto & $R \$ 3.850,00$ & $R \$ 10.594,13$ & $\mathrm{R} \$ 13.000,00$ \\
\hline Custo do pré-natal & $R \$ 1.000,00$ & $\mathrm{R} \$ 1.389,92$ & $\mathrm{R} \$ 13.000,00$ \\
\hline Custo da diária de UTI neonatal & $\mathrm{R} \$ 1.500,00$ & $\mathrm{R} \$ 2.000,00$ & $\mathrm{R} \$ 10.000,00$ \\
\hline$\%$ de uso de SET no mercado brasileiro & $16 \%$ & $20 \%$ & $24 \%$ \\
\hline Dias de internação - gravidez múltipla & 1,00 & 10,66 & 31,00 \\
\hline Dias de internação - gravidez única & 1,00 & 5,90 & 31,00 \\
\hline
\end{tabular}




\section{Análise de impacto orçamentário Cenário $\mathrm{A}$}

Em cinco anos, o reembolso de um ciclo de SET sequencial resultaria em um impacto de $\mathrm{R} \$ 34.104 .623 \mathrm{em}$ operadoras de grande porte, $\mathrm{R} \$ 3.513 .914$ em operadoras de médio porte e de $R \$ 579.000$ em operadoras de pequeno porte.

Considerando um cenário em que 30\% das pacientes estariam dispostas a pagar pelos medicamentos não reem- bolsados pelo sistema de saúde suplementar, o impacto orçamentário em cinco anos seria de $\mathrm{R} \$ 10.231 .387$ em operadoras de grande porte, de $\mathrm{R} \$ 1.054 .174$ em operadoras de médio porte e de $R \$ 173.700$ em operadoras de pequeno porte. Foi ainda possível estimar que o custo de economia por paciente foi de $\mathrm{R} \$$ 8.682. O resumo do impacto orçamental nos primeiros cincos anos é descrito na Figura 6 e o resumo dos cenários é descrito na Figura 7.

Tabela 4. Número médio de beneficiários e número total de mulheres entre 20 e 35 anos por porte de operadora

\begin{tabular}{|c|c|c|c|c|c|c|c|c|}
\hline Parâmetro & 2018 & 2019 & 2020 & 2021 & 2022 & 2023 & 2024 & 2025 \\
\hline $\begin{array}{l}\text { Número médio de } \\
\text { beneficiários - grande } \\
\text { porte }\end{array}$ & 455.435 & 459.170 & 462.935 & 466.731 & 470.558 & 474.417 & 478.307 & 482.229 \\
\hline $\begin{array}{l}\text { Número médio de } \\
\text { beneficiários - médio porte }\end{array}$ & 46.925 & 47.310 & 47.698 & 48.089 & 48.483 & 48.881 & 49.282 & 49.686 \\
\hline $\begin{array}{l}\text { Número médio de } \\
\text { beneficiários - pequeno } \\
\text { porte }\end{array}$ & 7.732 & 7.795 & 7.859 & 7.924 & 7.989 & 8.054 & 8.120 & 8.187 \\
\hline $\begin{array}{l}\text { Número de mulheres de } \\
20-35 \text { anos - grande porte }\end{array}$ & 59.481 & 59.301 & 59.141 & 59.078 & 59.035 & 59.019 & 59.015 & 58.995 \\
\hline $\begin{array}{l}\text { Número de mulheres de } \\
20-35 \text { anos - médio porte }\end{array}$ & 6.129 & 6.110 & 6.094 & 6.087 & 6.083 & 6.081 & 6.080 & 6.078 \\
\hline $\begin{array}{l}\text { Número de mulheres de } \\
20-35 \text { anos - pequeno porte }\end{array}$ & 1.010 & 1.007 & 1.004 & 1.003 & 1.002 & 1.002 & 1.002 & 1.002 \\
\hline
\end{tabular}

Tabela 5. População elegível para o tratamento com fertilização in vitro. Note que parte dos pacientes que não tiveram nascidos vivos transitam para o próximo ano. Os procedimentos reembolsados dependem do cenário em análise

\begin{tabular}{|c|c|c|c|c|c|c|c|}
\hline $\begin{array}{l}\text { Populaçã } \\
\text { pagador }\end{array}$ & Ano & 2021 & 2022 & 2023 & 2024 & 2025 & Total \\
\hline \multirow{4}{*}{$100 \%$} & Sistema de Saúde Suplementar & 141.046 & 209.598 & 243.486 & 260.795 & 270.679 & 1.125 .604 \\
\hline & Operadora de grande porte & 1.356 & 2.015 & 2.341 & 2.507 & 2.602 & 10.820 \\
\hline & Operadora de médio porte & 140 & 208 & 241 & 258 & 268 & 1.115 \\
\hline & Operadora de pequeno porte & 23 & 34 & 40 & 43 & 44 & 184 \\
\hline \multirow{4}{*}{$30 \%$} & Sistema de Saúde Suplementar & 42.314 & 62.879 & 73.046 & 78.238 & 81.204 & 337.681 \\
\hline & Operadora de grande porte & 407 & 604 & 702 & 752 & 781 & 3.246 \\
\hline & Operadora de médio porte & 42 & 62 & 72 & 77 & 80 & 334 \\
\hline & Operadora de pequeno porte & 7 & 10 & 12 & 13 & 13 & 55 \\
\hline
\end{tabular}

* Disposição para pagar custo não reembolsado.

Tabela 6. Número de pacientes reembolsadas, durante cinco anos, considerando 100\% ou 30\% de disposição de pagar pelos medicamentos não reembolsados e comparado com a projeção de utilização da fertilização in vitro no Brasil com crescimento anual de $20 \%$

\begin{tabular}{lccc}
\hline Pacientes tratadas (2021-2025) & Disposição para pagar 100\% & Disposição para pagar 30\% & Crescimento anual de 20\% \\
\hline Sistema de Saúde Suplementar & 704.633 & 211.390 & 81.672 \\
\hline Operadora de grande porte & 6.774 & 2.032 & 785 \\
\hline Operadora de médio porte & 698 & 209 & 81 \\
\hline Operadora de pequeno porte & 115 & 34 & 13 \\
\hline
\end{tabular}




\section{Cenário B}

Em cinco anos, o reembolso de dois ciclos de SET sequencial resultaria em um impacto de $\mathrm{R} \$ 50.261 .335 \mathrm{em}$ operadoras de grande porte, de $R \$ 5.178 .594$ em operadoras de médio porte e de $R \$ 853.295$ em operadoras de pequeno porte.

Considerando um cenário em que 30\% das pacientes estariam dispostas a pagar pelos medicamentos não reembolsados pelo sistema de saúde suplementar, o impacto orçamentário em cinco anos seria de R\$15.078.401 em operadoras de grande porte, de $\mathrm{R} \$ 1.553 .401 \mathrm{em}$ operadoras de médio porte e de R\$255.989 em operadoras de pequeno porte. Foi ainda possível estimar que o custo de economia por paciente foi de $\mathrm{R} \$ 12.795$.

\section{Cenário C}

Em cinco anos, o reembolso de um ciclo de SET sequencial resultaria em um impacto de $\mathrm{R} \$ 53.746 .245 \mathrm{em}$ operadoras de grande porte, de $R \$ 5.537 .656$ em operadoras de médio porte e de R\$912.459 em operadoras de pequeno porte. Considerando um cenário em que 30\% das pacientes estariam dispostas a pagar pelos medicamentos não reembolsados pelo sistema de saúde suplementar, o impacto orçamentário em cinco anos seria de R\$ 16.123 .874 em operadoras de grande porte, de R\$ 1.661.297 em operadoras de médio porte e de R\$273.738 em operadoras de pequeno porte. Foi ainda possível estimar que o custo de economia por paciente foi de R\$ 13.682.

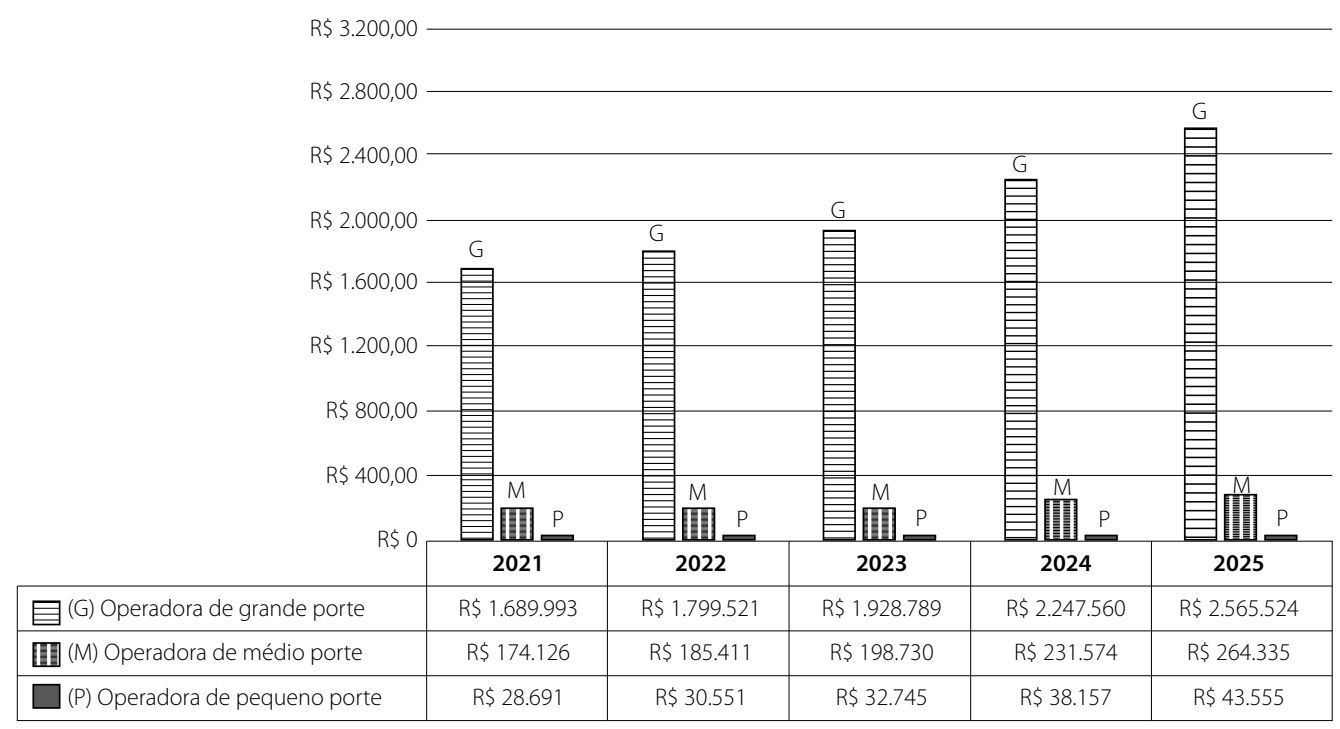

Figura 6. Impacto orçamental nos primeiros cinco anos do reembolso de 1 ciclo de SET sequencial por porte de operadora (Cenário A): grande porte $(\mathrm{G})$, médio porte $(\mathrm{M})$ e pequeno porte (P). Foi considerado um cenário no qual 30\% das pacientes estariam dispostas a pagar pelos medicamentos não reembolsados.

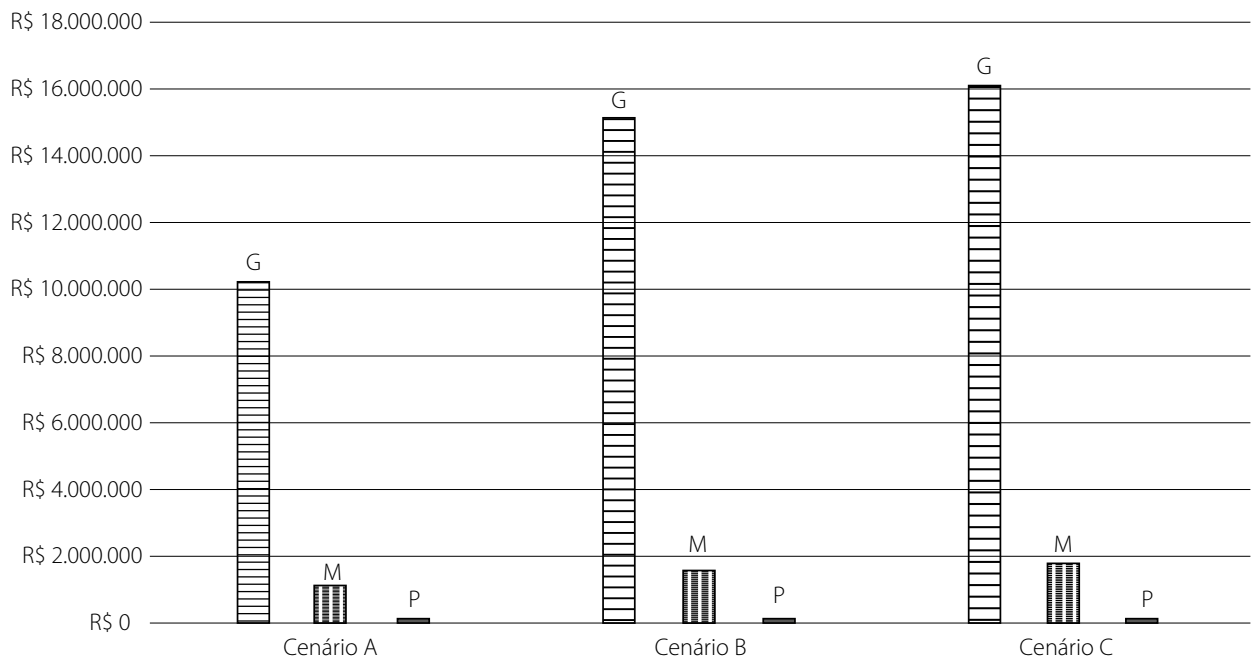

Figura 7. Impacto orçamental cumulativo de 5 anos (2021-2015) na implementação de cenários A, B e C em operadoras de grande porte $(G)$, médio porte $(M)$ e pequeno porte $(P)$, considerando uma disposição de pagar custo não reembolsado de $30 \%$. 


\section{Cenário D}

No cenário em que 30\% das pacientes elegíveis estariam dispostas a pagar pela totalidade do procedimento de fertilização in vitro (out-of pocket) - procedimento, honorários médicos e medicamento -, o aumento progressivo do uso de SET sequencial ao invés de DET acarretaria numa economia de R\$161.616.282 para o sistema de saúde privado brasileiro, em cinco anos.

\section{Projeção do crescimento e utilização da fertilização in vitro}

A partir da projeção (Figura 5), foi calculado que, entre 2021 e 2025, uma população de 77.588 pacientes com idade inferior ou igual a 35 anos de idade será tratada por infertilidade com o recurso da fertilização in vitro.

Assim, no cenário em que a utilização da fertilização in vitro no Brasil cresce em um ritmo de $20 \%$ anualmente, o aumento progressivo do uso de SET sequencial ao invés de DET acarretaria numa economia de $\mathrm{R} \$ 59.319 .276$ para o sistema de saúde privado brasileiro, em cinco anos.

\section{Análise de sensibilidade}

Na análise de sensibilidade, os parâmetros mais sensíveis foram a taxa de infertilidade, casais que procuram ajuda e custo do medicamento. Esses parâmetros estão diretamente ligados à população que utiliza o procedimento de fertilização in vitro ou ao custo do procedimento (Figura 8).

\section{Discussão}

Até onde temos conhecimento, este estudo consiste na primeira análise de impacto orçamentário avaliando um cenário de possível incorporação da técnica de fertilização in vitro com a implementação de SET sequencial no sistema de saúde privado brasileiro. Atualmente, a maioria das publicações disponíveis na literatura consiste em análises de custo-efetividade, que não refletem fielmente o impacto global no sistema de saúde, resultante da incorporação e reembolso de determinada tecnologia. Outros tipos de estudos atualmente disponíveis apenas comparam diretamente os custos inerentes a cada um dos procedimentos de interesse. Nesse cenário, observa-se uma lacuna de evidências que suportem e avaliem o impacto da incorporação de tecnologias no sistema como um todo.

O presente estudo consistiu em duas análises principais. A primeira comparou diretamente os custos do aumento progressivo da utilização do procedimento de fertilização in vitro com SET sequencial (SET + SET) em relação ao DET no cenário atual brasileiro (cenário D). Observou-se uma economia considerável de recursos do sistema privado brasileiro com essa abordagem, uma vez que, apesar dos custos de ambos os procedimentos de fertilização in vitro em si não serem reembolsados pelo sistema de saúde privado no cenário atual, os custos inerentes ao pré-natal, ao parto e às complicações são cobertos para pacientes seguradas. A economia de custos

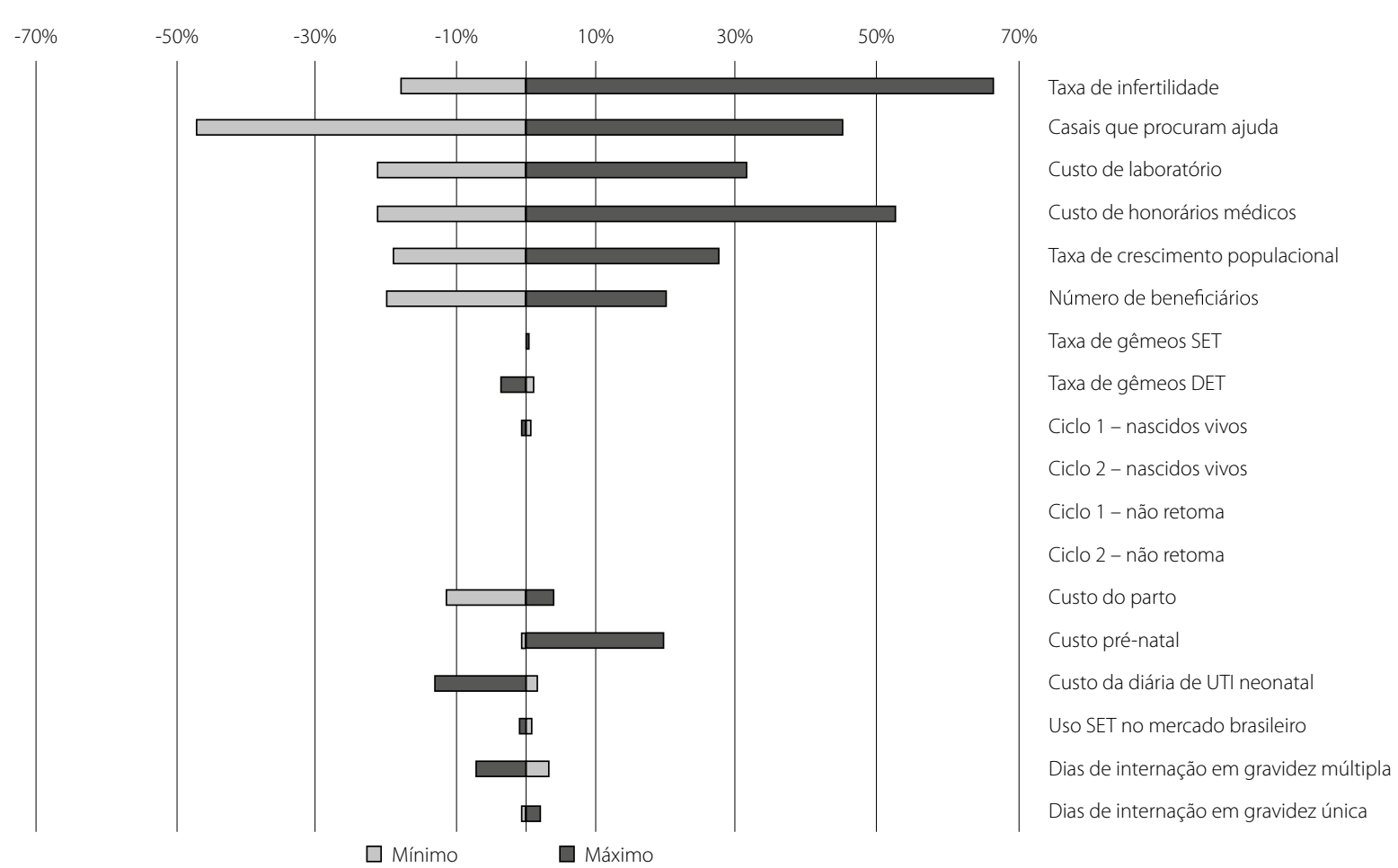

Figura 8. Resultado da Análise de Sensibilidade Determinística - Cenário A. 
detectada está relacionada a menores taxas de gemelaridade e, consequentemente, menos complicações atribuídas à técnica SET, uma vez que a gestação múltipla é responsável por maior despendimento de recursos por parte das operadoras de saúde do que o observado em gestações únicas.

É necessário destacar que a economia modelada do cenário D de R\$ 161 milhões é potencialmente sobredimensionada, visto que pressupõe um crescimento da utilização da fertilização in vitro excessiva e só é plausível para os cenários nos quais ocorre o reembolso/cobertura do procedimento pelos planos de saúde ou pelo estado. Assim, é plausível assumir que o valor real da economia seria entre $\mathrm{R} \$ 59$ milhões e R\$ 162 milhões. A economia de R\$ 59 milhões foi obtida considerando um crescimento anual de $20 \%$ da utilização atual do procedimento no Brasil.

A comparação direta dos custos dos procedimentos obtidos para o cenário brasileiro foi similar aos resultados obtidos para outros países, como, por exemplo, nos resultados reportados por Crawford et al. (2016), sob a perspectiva do pagador dos Estados Unidos. Entre 2012 e 2013, na comparação de custos referentes à utilização de um ciclo de DET vs. dois ciclos sequenciais de SET, em mulheres entre 19 e 45 anos, estimou-se uma diminuição de custos entre USD 195 e 386 milhões com o uso de SET sequenciais (Crawford et al., 2016). Outro estudo, publicado na forma de um abstract, estimou uma economia anual de custos de U\$D 110.395.255 num cenário de implantação de SET sequencial vs. DET (Klenov et al., 2017).

A grande maioria de estudos publicados avaliando o procedimento SET vs. DET é realizada em cenários nos quais o procedimento de fertilização in vitro não é reembolsado, visto que essa situação estimula a transferência múltipla de embriões com o objetivo de aumentar a chance de sucesso por ciclo e, consequentemente, tem o potencial de aumentar situações adversas durante a gravidez (Carpinello et al., 2016).

A segunda análise deste estudo avaliou a possível economia de recursos em cenários em que as operadoras de saúde reembolsassem também a técnica SET sequencial (procedimento, honorários médicos e, em uma das opções, medicamentos), além dos custos já normalmente cobertos em pacientes seguradas (pré-natal, parto e complicações). Observou-se que, em todos os cenários avaliados, o reembolso de SET sequencial (procedimento, honorários médicos e medicamentos) acarretaria um impacto incremental de cerca de R\$10 a 16 milhões em cinco anos, em operadoras de grande porte no cenário de 30\% de disposição para pagar pelos medicamentos não reembolsados pelo sistema de saúde privado. Tais resultados correspondem ao custo incremental de cerca de R\$ 8 a 13 mil por paciente elegível que recorreu ao procedimento de fertilização in vitro.

Ainda assim, foi possível demostrar que o custo de economia implementando a técnica de SET sequencial para as mulheres com idade inferior ou igual 35 é consideravelmente menor do que anteriormente se pensava, $\mathrm{R} \$ 8$ a 13 mil por paciente, considerando um custo de tratamento entre $R \$ 10$ e 20 mil por paciente.

É importante destacar que, em cenários nos quais a fertilização in vitro foi introduzida como um procedimento médico reembolsável por um estado ou país, o uso frequente de técnicas de SET foi demostrado como opção mais economicamente viável a longo prazo e, em certos casos, como o da Bélgica, por exemplo, a utilização de SET é obrigatória para que ocorra o reembolso (UZ Brussel Fertility Clinic CRG, s.d.; Vélez et al., 2014) ). Nesse caso em específico, a diminuição de embriões transferido por mulher, reduziu em $50 \%$ as gestações múltiplas e, consequentemente, diminuiu os custos associados (Gerris, 2007; Peeraer et al., 2014).

Esta análise de impacto orçamentário não está isenta de limitações metodológicas. Devido ao limitado número de publicações de dados e informações sobre procedimentos de fertilização in vitro, diversos dados de custo de procedimentos, honorários médicos e medicamentos foram obtidos a partir da opinião de especialistas, fontes que apresentam baixo grau de evidência e confiabilidade, assim como alta variabilidade, e podem estar subestimados. Por outro lado, optou-se por utilizar sempre que possível o cenário mais conservativo nas análises para os custos e uso de recursos de forma geral no modelo desenvolvido, logo esses valores podem estar superestimados. Por exemplo, a tendência mundial é de adiar a gravidez e, consequentemente, a procura por tratamentos de fertilidade, fazendo com que a implementação de SET sequencial para mulheres com idade $\leq 35$ possa refletir uma população que foi sobrestimada (Ministério da Saúde, 2014b).

Os custos resultantes de permanência na UTI neonatal, em que o tempo de permanência médio de gravidez múltipla é o dobro dos casos de gravidez única, foram possivelmente subestimados. Quando considerada literatura, algumas publicações relatam que a diferença entre o tempo de permanência na UTI neonatal nos dois grupos analisados - gravidez múltipla e gravidez única - é consideravelmente maior (Forman et al., 2014; Lemos et al., 2013). É importante relembrar que complicações de uma gravidez múltipla que possam surgir após a saída da maternidade não foram consideradas nesta modelação.

Em suma, observou-se que, considerando um cenário brasileiro de realização dos procedimentos de fertilização in vitro sem reembolso do valor deles (valor do procedimento com exames, medicamentos e honorários) pelas operadoras de saúde [reembolso apenas de pré-natal, parto e complicações pós-parto da gestante e do(s) recém-nascido(s)], o aumento progressivo do uso de SET sequencial vs. DET foi considerado menos oneroso para as operadoras. Entretanto, considerando um cenário de reembolso total do procedimento pelas operadoras de saúde (incluindo o custo do procedimento, honorários médicos e medicamentos), o 
resultado observado foi um custo incremental de cerca de $\mathrm{R} \$ 8$ a 13 mil por paciente para o sistema privado brasileiro.

\section{Conclusão}

A substituição do uso do procedimento DET pelo SET sequencial potencialmente fornece uma economia referente aos custos de pré-natal, parto e complicações pós-parto, considerando o cenário atual sem reembolso de procedimentos de fertilização in vitro. Considerando uma incorporação progressiva do procedimento SET sequencial no Sistema de Saúde Suplementar, o custo incremental foi estimado em $\mathrm{R} \$ 8$ a $13 \mathrm{mil} /$ paciente.

\section{Agradecimentos}

Agradecemos a Kantar Health (São Paulo, Brasil) pelo apoio técnico e diversas sugestões e críticas durante a construção do artigo.

\section{Referências bibliográficas}

ANS - Agência Nacional de Saúde Suplementar. Beneficiários de planos privados de saúde, por cobertura assistencial (Brasil - 2009-2019). 2018. Available from: https://www.ans.gov.br/perfil-do-setor/dados-gerais. Accessed on: July 30, 2019.

ANS - Agência Nacional de Saúde Suplementar. Procedimentos de concepção têm cobertura obrigatória pelos planos de saúde? 2019 Available from: http://www.ans.gov.br/aans/index.php?option=com_centraldeatendimento\&view=pergunta\&negativo $=1 \&$ categoriald $=186 \&$ resposta $=464 \&$ historico=558\&start=4. Accessed on: July 30, 2019.

ANS - Agência Nacional de Saúde Suplementar. Resolução normativa - RN n० 428, de 7 de novembro de 2017. Atualiza o Rol de Procedimentos e Eventos em Saúde, que constitui a referência básica para cobertura assistencial mínima nos planos privados de assistência à saúde, contratados a partir de 10 de janeiro de 1999; fixa as diretrizes de atenção à saúde; e revoga as Resoluções Normativas - RN n 387, de 28 de outubro de 2015, e RN no 407, de 3 de junho de 2016. 2017. Available from: http://www.ans.gov.br/component/legislacao/?view=legislacao\&task=TextoLei\&format=raw\&id=MzUwMg==. Accessed on: July 30, 2019.

Anvisa - Agência Nacional de Vigilância Sanitária. SisEmbrio - 120 Relatório do Sistema Nacional de Produção de Embriões. 2019. Available from: http://portal.anvisa.gov.br/documents/4048533/4994015/12\%C2\%BA+Relat\%C3\%B3rio+do+Sistema+Nacional+de+Produ\%C3\%A7\%C3\%A3o+de+Embri\%C3\%B5es+-+SisEmbrio.pdf/29f37c42-803d-4fe9-8f16-cf6cfc70f40e. Accessed on: July 30, 2019.

Blumenauer V, Czeromin U, Fehr D, Fiedler K, Gnoth C, Krüssel JS, et al. D.I.R-Annual 2017. J Reproduktionsmed Endokrinol. 2018;15(5-6):216-49.

Boivin J, Bunting L, Collins JA, Nygren KG. International estimates of infertility prevalence and treatment-seeking: potential need and demand for infertility medical care. Hum Reprod. 2007;22(6):1506-12.

Brasil. Lei no 9.656, de 3 de junho de 1998. Dispõe sobre os planos e seguros privados de assistência à saúde. 1998. Available from: http://www2. camara.leg.br/legin/fed/lei/1998/lei-9656-3-junho-1998-353439-publicacaooriginal-1-pl.html. Accessed on: July 30, 2019.

Brasil. Lei no 11.935, de 11 de maio de 2009. Altera o art. 36-C da Lei no 9.656, de 3 de junho de 1998, que dispõe sobre os planos e seguros privados de assistência à saúde. 2009. Available from: http://www.planalto.gov.br/ ccivil_03/LEIS/L9263.htm. Accessed on: July 30, 2019.
Carpinello OJ, Casson PR, Kuo CL, Raj RS, Sills ES, Jones CA. Cost Implications for Subsequent Perinatal Outcomes After IVF Stratified by Number of Embryos Transferred: A Five Year Analysis of Vermont Data. Appl Health Econ Health Policy. 2016;14(3):387-95.

Collucci C. Justiça obriga planos a pagarem por tratamento de reprodução assistida. São Paulo: Folha de São Paulo; 2017. Available from: https:// www1.folha.uol.com.br/equilibrioesaude/2017/03/1866912-justica-obrigada-planos-a-pagarem-por-tratamento-de-reproducao-assistida.shtml. Accessed on: July 30, 2019.

Conselho Federal de Medicina. Resolução CFM n 2.168, de 21/09/2017. 2017. Available from: https://flaviotartuce.jusbrasil.com.br/noticias/519764480/ resolucao-2168-2017-do-conselho-federal-de-medicina. Accessed on: July 30, 2019.

Correa MCDV, Loyola MA. Tecnologias de reprodução assistida no Brasil: opções para ampliar o acesso. Physis. 2015;25(3):753-77.

Crawford S, Boulet SL, Mneimneh AS, Perkins KM, Jamieson DJ, Zhang Y, et al. Costs of achieving live birth from assisted reproductive technology: a comparison of sequential single and double embryo transfer approaches. Fertil Steril. 2016;105(2):444-50.

Cui W. Mother or nothing: the agony of infertility. Bull World Health Organ. 2010;88(12):881-82.

Cutting R. Single embryo transfer for all. Best Pract Res Clin Obstet Gynaecol. 2018:53:30-7.

Datasus - Departamento de Informática do Sistema Único de Saúde do Brasil. 2018. Available from: http://www2.datasus.gov.br/DATASUS/index. php. Accessed on: July 30, 2019.

ESHRE. The ESHRE Capri Workshop Group, Social determinants of human reproduction. Hum Reproduct. 2001;16(7):1518-26.

Farquhar C, Marjoribanks J. Assisted reproductive technology: an overview of Cochrane Reviews. Cochrane Database Syst Rev. 2018;8:CD010537.

Forman EJ, Hong KH, Franasiak JM, Scott RT. Obstetrical and neonatal outcomes from the BEST Trial: Single embryo transfer with aneuploidy screening improves outcomes after in vitro fertilization without compromising delivery rates. Am J Obstet Gynecol. 2014;210(2):157.e1-6.

Gerris J. The near elimination of triplets in IVF. Reprod Biomed Online. 2007;15(3):40-4.

Grady R, Alavi N, Vale R, Khandwala M, McDonald SD. Elective single embryo transfer and perinatal outcomes: a systematic review and meta-analysis. Fertil Steril. 2012;97(2):324-31.

Henderson J, Hockley C, Petrou S, Goldacre M, Davidson L. Economic implications of multiple births: inpatient hospital costs in the first 5 years of life. Arch Dis Child Fetal Neonatal Ed. 2004;89(6):542-45.

Hernandez Torres E, Navarro-Espigares JL, Clavero A, López-Regalado ML, Camacho-Ballesta JA, Onieva-García M, et al. Economic evaluation of elective single-embryo transfer with subsequent single frozen embryo transfer in an in vitro fertilization/intracytoplasmic sperm injection program. Fertil Steril. 2015;103(3):699-706.

IBGE - Instituto Brasileiro de Geografia e Estatística. Diretoria de Pesquisas, Coordenação do Trabalho e Rendimento, Pesquisa Nacional por Amostra de Domicílios Contínua 2016-2017. 2017. Available from: https://www. ibge.gov.br/estatisticas/sociais/trabalho/9171-pesquisa-nacional-por-amostra-de-domicilios-continua-mensal.html. Accessed on: July 30, 2019.

IBGE - Instituto Brasileiro de Geografia e Estatística. IBGE divulga as Estimativas de População dos municípios para 2018. 2018. Available from: https://agenciadenoticias.ibge.gov.br/agencia-sala-de-imprensa/2013-agencia-de-noticias/releases/22374-ibge-divulga-as-estimativas-de-populacao-dos-municipios-para-2018. Accessed on: July 30, 2019.

Klenov V, Boulet S, Mejia R, Kissin DM, Van Voorhis B. Medical costs of live births from elective single embryo transfer vs double embryo transfer in donor oocyte cycles. Fertil Steril. 2017;108(3):e99. 
Kushnir VA, Barad DH, Albertini DF, Darmon SK, Gleicher N. Systematic review of worldwide trends in assisted reproductive technology 2004-2013. Reprod Biol Endocrinol. 2017;15(1):6.

Lee MS, Evans BT, Stern AD, Hornstein MD. Economic implications of the Society for Assisted Reproductive Technology embryo transfer guidelines: healthcare dollars saved by reducing iatrogenic triplets. Fertil Steril. 2016;106(1):189-195.e3.

Lemos EV, Zhang D, Van Voorhis BJ, Hu XH. Healthcare expenses associated with multiple vs singleton pregnancies in the United States. Am J Obstet Gynecol. 2013;209(6):586.e1-586.e11.

Malizia BA, Hacker MR, Penzias AS. Cumulative Live-Birth Rates after In Vitro Fertilization. N Engl J Med. 2009;360(3):236-43.

McLernon DJ, Harrild K, Bergh C, Davies MJ, de Neubourg D, Dumoulin JC, et al. Clinical effectiveness of elective single versus double embryo transfer: meta-analysis of individual patient data from randomised trials. BMJ. 2010;341:c6945.

Mehta VP, Patel JA, Gupta RH, Shah SI, Banker MR. One Plus One Is Better Than Two: Cumulative Reproductive Outcomes Are Better after Two Elective Single Blastocyst Embryo Transfers Compared to One Double Blastocyst Embryo Transfer. J Hum Reprod Sci. 2018;11(2):161-8.

Meldrum DR, Adashi EY, Garzo VG, Gleicher N, Parinaud J, Pinborg A, et al. Prevention of in vitro fertilization twins should focus on maximizing single embryo transfer versus twins are an acceptable complication of in vitro fertilization. Fertil Steril. 2018;109(2):223-9.

Ministério da Saúde. Análise de Impacto Orçamentário - Diretrizes Metodológicas. 2014a. Available from: https://portalarquivos2.saude.gov.br/ images/pdf/2014/novembro/10/Diretrizes-metodologicas-manual-de-analise-de-impacto-orcamentario-cienciasus.pdf. Accessed on: July 30 2019.

Ministério da Saúde. Estudo Saúde Brasil. Mais brasileiras esperam chegar aos 30 para ter primeiro filho. 2014b. Available from: http://www.brasil. gov.br/noticias/saude/2014/10/mais-brasileiras-esperam-chegar-aos-30-para-ter-primeiro-filho. Accessed on: July 30, 2019.

Monteleone PAA, Peregrino PFM, Baracat EC, Serafini PC. Transfer of 2 Embryos Using a Double-Embryo Transfer Protocol Versus 2 Sequential
Single-Embryo Transfers: The Impact on Multiple Pregnancy. Reprod Sci. 2018;25(10):1501-8.

Mukherjee GG, Khastgir G, Chattopadhyay R. Practical Guide in Andrology and Embryology. New Delhi: Jaypee Brothers Medical; 2018.

Pandian Z, Marjoribanks J, Ozturk O, Serour G, Bhattacharya S. Number of embryos for transfer following in vitro fertilisation or intra-cytoplasmic sperm injection. Cochrane Database Syst Rev. 2013;(7):CD003416.

Pantoja M, Fernandes A. Indications for in vitro fertilization at a public center for reproductive health in Campinas, Brazil. Int J Gynaecol Obstet. 2015;128(1):14-7.

Peeraer K, Debrock S, Laenen A, De Loecker P, Spiessens C, De Neubourg D, et al. The impact of legally restricted embryo transfer and reimbursement policy on cumulative delivery rate after treatment with assisted reproduction technology. Hum Reprod. 2014;29(2):267-75.

SART - Society for Assisted Reproductive Technology. What are my chances with ART? 2018. Available from: https://www.sartcorsonline.com/Predictor/Patient. Accessed on: July 30, 2019.

Sullivan SD, Mauskopf JA, Augustovski F, Jaime Caro J, Lee KM, Minchin M, et al. Budget impact analysis-principles of good practice: report of the ISPOR 2012 Budget Impact Analysis Good Practice II Task Force. Value Health. 2014;17(1):5-14.

UZ Brussel Fertility clinic CRG - Brussels (Jette) [homepage]. Financial aspects of an ART treatment [citado Out 22, 2019]. Available from: http:// www.brusselsivf.be/financial?doscroll=true\#NavL3.

Vélez MP, Connolly MP, Kadoch IJ, Phillips S, Bissonnette F. Universal coverage of IVF pays off. Hum Reprod. 2014;29(6):1313-9.

Woods B, Sideriis E, Palmer S, Latimer N, Soares M. NICE DSU technical support document 19: partitioned survival analysis for decision modelling in health care: a critical review. 2017. Available from: https://pure.york.ac.uk/ portal/en/publications/nice-dsu-technical-support-document-19(4abca204-a8a5-4880-9cce-190049aldaf9).html. Accessed on: Out 22, 2019.

Zegers-Hochschild F, Schwarze JE, Crosby JA, Musri C, Urbina MT. Assisted reproductive techniques in Latin America: the Latin American Registry 2016. Reprod Biomed Online. 2019;39(3). Available from: https://linkinghub.elsevier.com/retrieve/pii/S1472648319305292. 